Revue Française de Civilisation Britannique

XIV-1 | 2006

La dévolution des pouvoirs à l'Écosse et au Pays de Galles 1966-1999

\title{
Le Scottish National Party de 1979 à 1997 : anatomie d'un parti parvenu à la maturité politique
}

The Scottish National Party, 1979-1997: from wavering between moderation and extremism to reaching political maturity

\section{Annie Thiec}

\section{OpenEdition Journals}

Édition électronique

URL : http://journals.openedition.org/rfcb/1174

DOI : $10.4000 /$ rfcb. 1174

ISSN : 2429-4373

Éditeur

CRECIB - Centre de recherche et d'études en civilisation britannique

\section{Édition imprimée}

Date de publication : 2 janvier 2006

Pagination : 107-121

ISBN : 2-911580-23-0

ISSN : 0248-9015

\section{Référence électronique}

Annie Thiec, «Le Scottish National Party de 1979 à 1997 : anatomie d'un parti parvenu à la maturité politique », Revue Française de Civilisation Britannique [En ligne], XIV-1 | 2006, mis en ligne le 15 octobre 2016, consulté le 20 avril 2019. URL : http://journals.openedition.org/rfcb/1174 ; DOI : 10.4000/ rfcb. 1174

\section{(c) (i) $९$}

Revue française de civilisation britannique est mis à disposition selon les termes de la licence Creative Commons Attribution - Pas d'Utilisation Commerciale - Pas de Modification 4.0 International. 


\title{
Le Scottish National Party de 1979 à 1997 : anatomie d'un parti parvenu à la maturité politique
}

\author{
Annie THIEC \\ Université de Nantes
}

Le nouveau dispositif institutionnel mis en place à Édimbourg en 1999 dans le cadre du projet de dévolution à l'Écosse du gouvernement travailliste de Tony Blair est l'aboutissement d'un long processus de discussion et de coopération entamé à la fin des années 1970 entre les différents acteurs de la scène politique écossaise, partis politiques bien sûr mais aussi représentants de la société civile. Il s'agissait là du second projet travailliste de dévolution à l'Écosse, qui reçut l'approbation d'une très large majorité de la population écossaise lors du référendum du 11 septembre 1997, effaçant ainsi l'échec, dix-huit ans auparavant, du premier référendum sur la dévolution qui avait provoqué la chute du gouvernement de James Callaghan. L'Écosse dispose donc aujourd'hui d'un parlement — doté de pouvoirs législatifs — qui lui confère un important degré d'autonomie à l'intérieur du Royaume-Uni, au point que sur certaines questions, comme celle des droits d'inscription à l'université (tuition fees) par exemple, la nouvelle institution écossaise a mis en œuvre une politique différente de celle adoptée par le Parlement de Westminster pour l'Angleterre et le pays de Galles.

L'attachement des Écossais à l'idée de Home Rule n'est pas nouveau, puisque plusieurs organisations nationalistes, dont les revendications allaient d'une plus grande autonomie à l'indépendance totale, virent le jour en Écosse ${ }^{1}$ entre 1886, date de la création de la première Scottish Home Rule Association, et 1927, lorsque fut fondée l'association des étudiants nationalistes de Glasgow, The Glasgow University Scottish Nationalist Association, qui joua un rôle fondamental dans la création du premier parti nationaliste écossais, National Party of Scotland, en 1928. Idéologiquement à gauche sur l'échiquier politique écossais, le NPS était majoritairement composé de militants issus de l'aile modérée du mouvement nationaliste mais il comptait également dans ses rangs des partisans de l'indépendance pure et simple de l'Écosse, notamment parmi les anciens membres de la Scots National League et du Scottish National Movement. Quatre ans plus tard un second parti nationaliste du nom de Scottish Party (SP) faisait son apparition sur la scène politique écossaise, fondé quant à lui par des dissidents de la section du

\footnotetext{
${ }^{1}$ La toute première organisation nationaliste écossaise, d'allégeance plutôt conservatrice, The National Association for the Vindication of Scottish Rights, fut fondée en 1853, mais elle disparut trois ans plus tard.
} 
Parti unioniste ${ }^{2}$ de Glasgow Cathcart. Les mauvais résultats électoraux obtenus par le NPS eurent tôt fait d'exacerber les divergences idéologiques entre modérés et partisans de l'indépendance qui se soldèrent par l'exclusion des membres les plus radicaux, permettant ainsi la fusion des deux partis nationalistes au mois d'avril 1934 pour former le Scottish National Party.

Après une victoire de courte durée lors de l'élection partielle de Motherwell and Wishaw le 12 avril $1945^{3}$, qui donna au SNP son premier député, le Parti nationaliste dut attendre le début des années $1960^{4}$ pour enregistrer un net progrès sur la scène politique en termes à la fois de recrutement et de résultats électoraux ${ }^{5}$. Mais la véritable percée politique du SNP se produisit à la fin de la décennie, dans un contexte d'inflation croissante que le gouvernement travailliste ne parvenait pas à maîtriser et alors que l'Écosse subissait de plein fouet la récession qui frappait le secteur de l'industrie lourde. Après une nette progression aux élections législatives de $1966^{6}$, la victoire de la candidate nationaliste Winifred Ewing à l'élection partielle de Hamilton le 2 novembre 1967, avec $46 \%$ des suffrages contre 41,5\% pour le candidat travailliste, sonnait comme un avertissement lancé au Parti travailliste, avertissement renouvelé lors des élections locales de l'année suivante, à l'issue desquelles le SNP, qui avait recueilli $30,1 \%$ des suffrages, comptait désormais 107 conseillers élus ${ }^{7}$.

La progression du SNP sur la scène politique écossaise, et de Plaid Cymru au pays de Galles ${ }^{8}$, perçue comme une menace réelle par le gouvernement travailliste, fut à l'origine de l'annonce au mois de décembre 1968 de la mise en place d'une Commission Royale sur la Constitution chargée d'étudier les rapports entre les différentes composantes de Royaume-Uni. ${ }^{9}$ La Commission Kilbrandon, du nom de

\footnotetext{
${ }^{2}$ Le Parti conservateur écossais se nommait à l'époque Scottish Unionist Association. C'est en 1965 qu'il s'est adjoint le qualificatif de «conservateur», devenant alors Scottish Conservative and Unionist Association.

${ }^{3}$ Le SNP perdit le siège aux élections législatives de juillet 1945.

${ }^{4}$ Lors de l'élection partielle de Glasgow Bridgeton, le 10 novembre 1961, le candidat nationaliste recueillit $18,7 \%$ des voix, arrivant ainsi en troisième position derrière les candidats travailliste et conservateur. Il convient de préciser toutefois que le Parti libéral ne présentait pas de candidat pour cette élection, ce qui profita incontestablement au SNP. Les résultats furent meilleurs encore l'année suivante lors de l'élection partielle de West Lothian, le 14 juin 1962, dans laquelle le candidat nationaliste, avec 23,3\% des voix, soit le double du pourcentage recueilli part le candidat conservateur $(11,4 \%)$ arrivé en troisième position, obtint la deuxième place derrière le travailliste $(50,9 \%)$.

${ }^{5}$ Sur les progrès du SNP au début des années 1960, voir entre autres Jacques LERUEZ, L'Écosse - Vieille Nation, Jeune État, Crozon : Editions Armeline, 2000, pp. 162-164 et Peter LYNCH, SNP - The History of the Scottish National Party, Cardiff: Welsh Academic Press, 2002, pp. 93-118.

${ }^{6}$ Le SNP était arrivé en deuxième position derrière le Parti travailliste dans trois des 23 circonscriptions dans lesquelles il présentait un candidat.

${ }^{7}$ Peter LYNCH, op.cit., p. 118.

${ }^{8}$ Le Parti nationaliste gallois avait de son côté remporté l'élection partielle de Carmarthen le 14 juillet 1966, recueillant $39 \%$ des suffrages contre $33 \%$ pour le Parti travailliste qui détenait précédemment le siège aux Communes.

${ }^{9}$ Edward Heath, quant à lui, annonça devant le congrès écossais du Parti conservateur réuni à Perth au mois de mai 1968 la mise en place d'un comité constitutionnel présidé par Sir Alec
} 
son second président, nommé à la suite du décès de Lord Crowther, rendit son rapport le 31 octobre $1973^{10}$. Entre-temps, le Parti travailliste avait perdu les élections législatives de 1970, et le gouvernement conservateur d'Edward Heath avait d'autres priorités, notamment l'adhésion du Royaume-Uni au Marché Commun et les relations entre employeurs et salariés. La découverte des gisements de pétrole en mer du Nord en 1971 allait cependant donner aux nationalistes un argument économique de poids : l'indépendance devenait désormais synonyme de prospérité économique. De fait, le conflit entre le gouvernement et les mineurs, le début de la quatrième guerre israélo-arabe au mois d'octobre 1973, provoquant la flambée du prix du pétrole et faisant par la même occasion des gisements de la mer du Nord un véritable atout économique, ainsi que la publication du rapport Kilbrandon jouèrent en faveur des nationalistes écossais à l'approche des élections législatives de 1974.

Il fallut cependant attendre la fin de la décennie avant que le gouvernement travailliste de James Callaghan ${ }^{11}$ parvînt finalement à surmonter les divisions au sein de son propre parti sur la question de la dévolution et à faire adopter par le Parlement britannique le 22 février 1978 ses deux projets d'assemblée pour l'Écosse et le pays de Galles. La « conversion » des travaillistes à la dévolution représentait à la fois une aubaine et un piège pour les nationalistes : en effet, la question du statut de l'Écosse au sein du Royaume-Uni était désormais à l'ordre du jour, même s'il ne s'agissait que d'accorder davantage d'autonomie à l'Écosse et non l'indépendance, comme le souhaitait le SNP. En revanche, le parti était contraint de prendre position par rapport au projet travailliste d'assemblée.

Or, depuis que la progression des nationalistes en Écosse et au pays de Galles avait mis la question de la dévolution à l'ordre du jour, le SNP oscillait entre modération et extrémisme quant à la stratégie à adopter pour atteindre son objectif politique. Ainsi, sa décision de soutenir finalement le projet travailliste de dévolution reposait sur un consensus fragile ${ }^{12}$ qui ne manqua pas de voler en éclats

Douglas-Home dont la mission était de faire rapidement des propositions de réforme visant à améliorer la façon dont l'Écosse était gouvernée. Le comité publia son rapport intitulé Scotland's Government au mois de mars 1970 : il recommandait la création d'une Convention écossaise de 125 membres élus au suffrage universel, dont les pouvoirs étaient analogues à ceux des commissions permanentes à Westminster et du Scottish Grand Committee.

${ }^{10}$ La Commission n'étant pas parvenue à un accord unanime, deux rapports furent publiés. Le rapport majoritaire, signé par onze des treize membres de la Commission, recommandait la dévolution législative pour l'Écosse et le pays de Galles, mais ne prévoyait pas d'assemblées régionales pour l'Angleterre. Le rapport minoritaire (Memorandum of Dissent), rédigé par les deux autres membres, stipulait qu'il fallait prévoir des assemblées régionales en Angleterre, même s'il n'y avait pas de parti nationaliste anglais. Les treize membres de la Commission rejetaient unanimement cependant le fédéralisme et le séparatisme, de même qu'ils étaient d'accord sur la mise en place d'une assemblée écossaise élue au suffrage universel direct et au scrutin proportionnel.

${ }^{11}$ Harold Wilson démissionna de ses fonctions de Premier ministre au mois de mars 1976 et fut remplacé par James Callaghan.

${ }^{12}$ Le SNP promettait d'approuver le projet d'assemblée du gouvernement, même s'il le jugeait loin d'être satisfaisant, mais dans le même temps il entendait ne laisser aucun doute sur son véritable objectif politique. 
après la victoire du Parti conservateur aux élections de mai 1979, plongeant dans le même temps le SNP dans une longue période d'introspection jusqu'à la fin des années 1980. Les résultats des élections législatives de 1987 et l'impopularité des réformes mises en œuvre en Écosse par Margaret Thatcher au cours de son troisième mandat permirent alors au SNP de faire avancer la cause de l'indépendance, poussant le Parti travailliste à s'engager à nouveau fermement sur la voie de la dévolution. Enfin, sous l'impulsion d'Alex Salmond, élu à la tête du parti à l'automne 1990, le SNP parvint à trouver la cohésion idéologique qui lui avait tant fait défaut vingt ans auparavant. Engagé désormais sur la voie de la modération et du pragmatisme, le parti put alors, sans se compromettre, participer à la promotion du projet de dévolution aux côtés des travaillistes et des libéraux-démocrates au cours de la campagne pour le référendum du 11 septembre 1997.

\section{Le SNP et la dévolution avant 1979 : entre modération et extrémisme}

Le mouvement nationaliste en Écosse a de tout temps été composé de groupes qui ne partageaient pas tous la même idéologie. La fusion en 1934 entre les deux partis nationalistes, NPS et SP, qui donna naissance au SNP, ne fit pas disparaître les dissensions qui existaient entre les deux partis d'une part, et à l'intérieur même du NPS d'autre part, entre des nationalistes modérés qui souhaitaient voir l'Écosse jouir d'une plus grande autonomie au sein du Royaume-Uni et les partisans de l'indépendance à tout prix.

Les divergences de vue sur la question de la stratégie à adopter pour atteindre cet objectif politique d'indépendance ont pu parfois être mises de côté au nom de l'intérêt supérieur du parti, mais face aux propositions du Parti travailliste dans les années 1970, le SNP a oscillé entre modération et extrémisme. Les gradualistes, favorables à la dévolution, certains d'entre eux montrant même peu d'enthousiasme pour l'indépendance, étaient également favorables à l'idée d'une coopération avec les autres partis politiques sur la question de la dévolution. Les fondamentalistes, quant à eux, étaient hostiles à la dévolution, qu'ils considéraient être un obstacle sur la voie de l'indépendance ${ }^{13}$. Il n'était d'ailleurs pas urgent pour le SNP de définir précisément sa position sur la question de la dévolution tant que la Commission Royale sur la constitution n'avait pas rendu son rapport.

Certes, le congrès annuel du parti de 1972 avait adopté une résolution par laquelle il s'engageait à accepter toutes les propositions de dévolution émanant de Westminster comme autant de pas en avant vers l'indépendance, tout en affirmant que l'objectif final du SNP restait l'indépendance. Les propositions contenues dans le rapport Kilbrandon, qui recommandait la mise en place d'une assemblée écossaise dont les attributions couvraient à peu près celles du Scottish Office, constituaient un projet de dévolution que le SNP pouvait donc soutenir. Pourtant, le comité exécutif national (National Executive Committee) du parti adopta une résolution exhortant la confédération des syndicats écossais à se joindre aux nationalistes pour demander

${ }^{13}$ Voir sur ce point James MITCHELL, Strategies for Self-Government, Edinburgh: Polygon, 1996, pp. 212-213. 
l'extension des pouvoirs économiques et financiers de l'assemblée. En réalité, tant qu'aucun parti au gouvernement ne faisait de propositions en matière de dévolution, le SNP pouvait se consacrer à faire progresser la cause de l'indépendance sans devoir se prononcer sur la position qu'il adopterait face à un projet d'assemblée. Cependant, dès lors que le nouveau gouvernement travailliste s'engagea à mettre en place une assemblée en Écosse, au lendemain des élections législatives de février 1974, il devint urgent pour les nationalistes de définir clairement leur position sur la question de la dévolution.

Toutefois, l'organisation du SNP, qui a la particularité d'être très décentralisée, ne favorisait pas la cohésion : si le congrès annuel avait pour vocation de débattre de la politique du parti, le conseil national (National Council), qui se réunissait trois fois par an, décidait quant à lui en dernier ressort du programme du parti, mais c'était le comité exécutif national (NEC), composé de 24 membres élus soit par le congrès annuel soit par le conseil national, qui dirigeait véritablement le parti ${ }^{14}$. En outre, à partir du mois de février 1974, non seulement le SNP se vit investi d'un nouveau rôle au Parlement, le gouvernement travailliste ayant besoin du soutien des libéraux et des nationalistes pour gouverner, mais les instances dirigeantes du parti, dont le numéro un en Écosse, qui n'était pas élu à Westminster, allaient désormais devoir compter pour la première fois dans l'histoire du SNP avec un groupe parlementaire, fort de sept membres dans un premier temps, puis de onze à partir d'octobre, qui durant les débats sur les projets de loi gouvernementaux sur la dévolution ne suivirent pas toujours les décisions du NEC.

Or, l'analyse par le parti lui-même des résultats du scrutin d'octobre mit en évidence un désaccord idéologique entre les sociaux-démocrates et l'aile traditionaliste, qui allait poursuivre le parti jusqu'au référendum de 1979 et bien audelà, jusqu'au début des années 1990. Les premiers étaient convaincus en effet que pour progresser le SNP devait conquérir une partie de l'électorat travailliste; ils étaient donc favorables à une ouverture à gauche du parti. Les traditionalistes, à l'inverse, estimaient que le nationalisme était au-dessus de toute idéologie, qu'elle soit de gauche ou de droite, et que la raison d'être du parti était le combat pour l'indépendance de l'Écosse. Selon le rapport présenté au conseil national de décembre 1974 par le numéro un du parti, William Wolfe, lui même socialdémocrate, le Parti travailliste avait progressé dans 30 des 71 circonscriptions écossaises entre le mois de février et le mois d'octobre ; il était donc essentiel pour le SNP de gagner la confiance des électeurs travaillistes plus enclins à soutenir la dévolution que les électeurs conservateurs. Une telle stratégie supposait l'adoption d'une position proche de celle du gouvernement sur la dévolution.

Aussi, lorsqu'au mois de mai 1975 le conseil national du parti approuva à une très large majorité une résolution du NEC selon laquelle le parti acceptait de prendre part à une assemblée écossaise quelle qu'elle fût, du moment qu'elle était démocratiquement élue, l'approche gradualiste semblait bel et bien avoir pris le pas

\footnotetext{
${ }^{14}$ Certaines fonctions au sein du comité sont plus importantes que d'autres. Ainsi le président (president) du parti a une fonction purement honorifique; le numéro un du parti avait jusqu'en 1988 le titre de chairman, qui a été remplacé ensuite par celui de convener. Il convient d'ajouter que le chairman n'était pas nécessairement élu au Parlement britannique.
} 
sur le radicalisme des partisans de l'indépendance à tout prix. Par ailleurs, le parti se donnait pour objectif d'obtenir l'extension des pouvoirs de l'assemblée jusqu'à ce qu'elle devienne un véritable parlement écossais à même de servir les intérêts du peuple écossais, ce qui signifiait que l'indépendance pouvait être atteinte de façon progressive. Un an plus tard, cependant, le congrès annuel votait une résolution qui cette fois indiquait un durcissement de la position du parti sur la dévolution : Le SNP rappelait tout d'abord que son objectif final était bel et bien l'indépendance et que même si le parti était prêt à accepter une assemblée dotée de pouvoirs limités comme une étape possible sur la voie de l'indépendance, seule l'indépendance pouvait répondre aux besoins des Écossais ${ }^{15}$. Il s'en était même fallu de peu pour qu'un amendement ne vînt mettre fin à l'engagement du parti à soutenir la mise en place d'une assemblée ${ }^{16}$.

Malgré l'insistance de William Wolfe sur la nécessité de rassurer les électeurs que la perspective de l'indépendance effrayait, le congrès de 1977 consacra la politique intransigeante de «l'indépendance, rien de moins» (Independence, Nothing Less). Aussi, en dépit de la décision du conseil national du parti, au mois de juin 1978, de faire campagne pour le Oui lors du référendum sur le Scotland Act, les divergences de vue entre le NEC, et notamment le numéro un du parti, et les élus à Westminster se firent fréquemment sentir. Ainsi Gordon Wilson, ancien secrétaire national du parti et élu depuis février 1974 dans la circonscription de Dundee East, demanda même expressément aux nationalistes de ne pas s'associer à l'adversaire de toujours, en l'occurrence le Parti travailliste, pendant la campagne du référendum. Le numéro un du parti, quant à lui, estimait qu'il était indispensable de soutenir les propositions du gouvernement, tout en essayant de rassembler au sein d'une nouvelle coalition tous ceux qui partageaient les objectifs du SNP, et notamment les membres du Scottish Labour Party fondé en janvier 1976 par le député travailliste écossais Jim Sillars ${ }^{17}$. Aussi, le NEC enjoignit-il les députés nationalistes de voter contre la motion de censure déposée à l'automne 1978 contre le gouvernement de James Callaghan $^{18}$, afin de soutenir ce dernier jusqu'au référendum. Cependant, neuf des onze députés nationalistes ne suivirent pas les consignes du NEC et votèrent avec l'opposition conservatrice ${ }^{19}$.

\footnotetext{
${ }^{15}$ Résolution $48 \mathrm{du} 42^{\mathrm{e}}$ congrès annuel du SNP, 29 mai 1976, dont le texte est cité dans Peter LYNCH, op.cit, pp. 147-148.

${ }^{16} \mathrm{~L}$ 'amendement en question proposait en effet d'exclure du texte de la résolution 48 la phrase «bien que prêt à accepter une assemblée aux pouvoirs limités comme une possible étape ». Il fut rejeté par 594 voix contre 425.

${ }^{17}$ Converti depuis peu à la cause de la dévolution, Jim Sillars estimait que les propositions travaillistes contenues dans le livre blanc de 1975 étaient insuffisantes. L'expérience du Scottish Labour Party fut de courte durée cependant puisque le parti cessa d'exister après la défaite de ses deux députés, dont Jim Sillars, aux élections législatives de 1979. Après avoir été l'ennemi des nationalistes, ce qui lui avait valu le surnom de 'Hammer of the Nats' à la fin des années 1960, Jim Sillars rejoignit finalement le SNP au mois de mai 1980. Il fut le principal architecte de la politique de l'indépendance en Europe défendue par le SNP à partir de la fin des années 1980.

${ }^{18}$ Le pacte libéral-travailliste était arrivé à échéance au mois de septembre 1978 ; le gouvernement travailliste était donc à nouveau minoritaire aux Communes.

${ }^{19}$ Seul George Reid vota contre la motion, tandis que Hamish Watt s'abstint. Le vote sur cette motion ne suffit pas toutefois à renverser le gouvernement.
} 
À la veille de la campagne du référendum, le SNP était donc pour le moins affaibli par le manque de cohésion dont il avait fait preuve tout au long des débats sur les propositions de dévolution. Aussi n'est-il guère surprenant que ces divergences d'opinion entre gradualistes et fondamentalistes aient atteint leur paroxysme après l'échec du référendum. Au lendemain du référendum, les nationalistes étaient satisfaits du résultat obtenu, puisque la majorité des votants s'était prononcée en faveur de la dévolution, même si cette majorité n'atteignait pas les $40 \%$ imposés par l'amendement Cunningham. Ils demandèrent alors au gouvernement de pourvoir dans les plus brefs délais à la mise en place de l'assemblée écossaise prévue par le Scotland Act. Lorsqu'il apparut que le gouvernement n'allait pas s'engager en ce sens, les députés nationalistes déposèrent une motion de censure contre le gouvernement. Le Parti conservateur ayant de son côté également déposé une motion de même nature, le gouvernement de James Callaghan fut bel et bien renversé le 28 mars 1979, par 311 voix contre 310.

\section{De l'introspection à l'amorce d'un renouveau}

Au lendemain du référendum du $1^{\mathrm{er}}$ mars, et plus encore à l'issue des élections législatives du 3 mai 1979, le SNP apparaissait comme le grand perdant de ces deux importantes consultations populaires qui auraient pu pourtant consacrer 1979 comme une année charnière dans l'histoire de l'Écosse, et le SNP comme le catalyseur de ce bouleversement; il ne faisait en effet aucun doute que la progression des nationalistes sur la scène politique avait été la raison principale de la conversion du Parti travailliste à la dévolution.

Les élections législatives de 1979 furent catastrophiques pour le SNP. Avec $17,3 \%$ des suffrages seulement, ce qui représentait une baisse de 13 points par rapport aux élections d'octobre 1974, il avait perdu neuf des onze sièges qu'il détenait et ne comptait plus désormais que deux députés à Westminster ${ }^{20}$. L'échec de la dévolution semblait donner raison aux fondamentalistes, qui reprochaient au parti d'avoir été trop conciliant avec le gouvernement, dont la proposition de dévolution n'était à leurs yeux qu'un moyen habile d'apaiser l'ardeur du sentiment national en Écosse. Aussi le SNP devait-il abandonner selon eux l'idée de la dévolution et prendre ses distances par rapport à Westminster. Mais les mécontentements se faisaient entendre également parmi les modérés, car le retour des conservateurs au pouvoir à Londres signifiait que la dévolution ne serait plus à l'ordre du jour pour les années à venir. De fait, les dévolutionnistes critiquaient le manque d'engagement réel du SNP sur le projet d'assemblée écossaise, tout particulièrement pendant la campagne du référendum.

Par ailleurs, les résultats des élections mettaient en évidence le dilemme auquel le SNP était toujours confronté sur le plan idéologique : sur les neuf sièges perdus,

${ }^{20}$ En outre, après être devenu le deuxième parti en Écosse en termes de pourcentage de voix recueillies $(30,4 \%)$ lors des élections d'octobre 1974, au cours desquelles les candidats nationalistes étaient arrivés en deuxième position dans 42 circonscriptions dont 35 détenues par le Parti travailliste, il s'effaçait à nouveau derrière le Parti conservateur et n'occupait plus la deuxième place que dans douze circonscriptions. 
en effet, sept avaient été reconquis par le Parti conservateur et deux par le Parti travailliste. Le SNP semblait donc être identifié parfois comme un parti de gauche et parfois à l'inverse comme un parti de droite. Le fait que de 1974 à 1979 les députés nationalistes aient tantôt voté avec le Parti travailliste au gouvernement, tantôt avec le Parti conservateur dans l'opposition, qui selon les instances dirigeantes du parti était la preuve au moins que le SNP n'était à la solde d'aucun des deux partis alternant au pouvoir à Londres, avait sans aucun doute largement contribué au manque de clarté manifeste sur le positionnement du SNP sur l'échiquier politique écossais. Les députés nationalistes écossais avaient même davantage voté avec les conservateurs au cours de trois des cinq sessions parlementaires qui s'étaient déroulées entre le mois d'octobre 1974 et le mois de mai 1979, ce qui apportait du crédit aux accusations lancées par les travaillistes qui qualifiaient les nationalistes écossais de 'Tartan Tories'.

De fait, la plupart des candidats nationalistes victorieux en 1974 avaient été élus dans des circonscriptions rurales précédemment représentées à Westminster par un député conservateur et mettaient en avant cet état de fait pour justifier leur opposition au gouvernement travailliste ${ }^{21}$. À l'inverse, les candidats qui s'étaient présentés, sans succès, dans des fiefs travaillistes, dans le bassin de Glasgow notamment, dont plusieurs des membres du NEC, voyaient d'un mauvais œil tout rapprochement avec le Parti conservateur à Westminster, qui ne pouvait qu'éloigner du SNP l'électorat travailliste que le parti devait selon eux à tout prix conquérir pour progresser sur la scène politique. C'est dans ce contexte d'un manque de cohésion idéologique qu'une nouvelle faction vit le jour au sein du SNP entre les événements du printemps 1979 et le congrès annuel de l'automne : il s'agissait essentiellement de gradualistes qui souhaitaient une réorientation à gauche du parti ${ }^{22}$. Par ailleurs, ils étaient favorables à des actions directes, pour protester notamment contre les fermetures d'usines et contre les licenciements qui en résultaient.

De leur côté, les fondamentalistes réclamaient une redéfinition des objectifs politiques du parti, qui fut effective, du reste, après le congrès de septembre 1979. Ainsi l'indépendance allait redevenir le thème principal des campagnes à venir du parti, qu'il s'agisse d'élections législatives générales ou partielles. Par ailleurs, le parti annonçait qu'il ne s'engagerait plus dans aucune transaction portant sur des projets de dévolution quels qu'ils fussent. Cette réorientation politique s' accompagna d'un renouvellement des cadres du parti : ainsi Gordon Wilson, fut élu chairman, en réaction contre la politique du parti jugée trop modérée entre 1974 et 1979, mais également pour neutraliser l'aile gauche du parti incarnée par le Groupe 79. Ce dernier ne parvint pas à conquérir la majorité des sièges au Comité National Exécutif du parti, mais lorsque le congrès du mois de mai 1981 adopta une résolution appelant à une campagne de désobéissance civile afin de protester contre la montée du chômage en Écosse, il crut y voir le signe de son influence sur la politique du parti. Cependant, suite à l'échec de cette campagne, qui se résuma finalement à une seule action à Édimbourg, le Groupe 79 fut dissous et sept de ses

\footnotetext{
${ }^{21}$ Les députés nationalistes avaient par exemple voté contre la nationalisation des chantiers navals en 1976.

${ }^{22}$ Lors de son meeting inaugural au mois d'août 1979, le «Groupe 79 » avait défini trois priorités : l'indépendance, le socialisme et le républicanisme.
} 
membres exclus du SNP au mois de septembre 1982, parmi lesquels le futur leader du parti, Alex Salmond ${ }^{23}$. Au lendemain de la dissolution du Groupe 79, Gordon Wilson dans son rapport au Conseil National du parti, reconnaissait que depuis l'échec du référendum sur la dévolution, le SNP avait été dominé par des guerres intestines au point d'en négliger son objectif principal, à savoir la liberté nationale ${ }^{24}$.

Les élections législatives de 1983 confirmèrent le déclin amorcé en $1979^{25}$, mais ce nouveau revers conduisit cette fois le parti à redéfinir sa politique générale et à rechercher une position consensuelle sur la question de l'avenir constitutionnel de l'Écosse. En effet, Gordon Wilson, pourtant élu à la tête du parti en 1979 sur une plate-forme fondamentaliste, était désormais convaincu qu'il était essentiel et urgent de ramener le parti à une position plus modérée. Ainsi, il parvint à aplanir les dissensions internes sur l'Europe et sur la dévolution ${ }^{26}$. Dès le congrès annuel de 1983, le parti, autrefois hostile à la construction européenne $e^{27}$, se prononça en faveur de l'adhésion à la CEE de l'Écosse une fois indépendante ${ }^{28}$. L'Europe était du reste un domaine sur lequel le consensus était possible entre gradualistes et fondamentalistes, et entre sociaux-démocrates et défenseurs de la neutralité idéologique du parti. Par ailleurs, Gordon Wilson tenta de réconcilier fondamentalistes et gradualistes autour d'un projet de convention constitutionnelle élue à la proportionnelle qui aurait pour mission de déterminer le statut constitutionnel de l'Écosse. Cette idée, rejetée dans un premier temps, fut finalement adoptée par le congrès du parti en septembre 1984. Cette proposition présentait l'avantage de permettre au SNP de faire campagne pour l'indépendance sans rejeter toutefois la dévolution. Par ailleurs, le parti n'excluait pas la coopération avec d'autres partis politiques, notamment au sein de l'association dévolutionniste, Campaign for a Scottish Assembly (CSA), qui avait été créée au lendemain des élections législatives de 1979 par quelques militants, travaillistes et nationalistes, dévoués à la cause de la dévolution.

Les effets de cette nouvelle politique du consensus ne tardèrent pas à se faire sentir puisque le SNP obtint des résultats encourageants aux élections locales puis au élections européennes de 1984, ainsi qu'aux élections régionales de 1986. C'est seulement au lendemain des élections législatives de 1987, cependant, que le contexte politique permit au SNP de s'affirmer à nouveau sur la scène politique écossaise. Les élections législatives du 10 juin 1987 furent en effet désastreuses pour le Parti conservateur en Écosse qui, en ne recueillant que $24 \%$ des suffrages, soit $4,4 \%$ de moins qu'en 1983, perdait onze des vingt-et-un sièges qu'il détenait précédemment. Surtout, plus des trois-quarts des suffrages exprimés s'étaient portés sur un parti autre que celui appelé à gouverner à nouveau l'Écosse, le seul parti, en

${ }^{23}$ Il fut réintégré par la suite.

${ }^{24}$ Rapport du chairman au Conseil National du Scottish National Party du 4 décembre 1982. Voir à ce sujet Roger LEVY, op. cit., p. 105.

${ }^{25}$ Le SNP ne recueillit que $11,8 \%$ des suffrages, accusant ainsi un recul de 5,5 points encore par rapport à 1979 et perdant 53 des 72 cautions électorales versées.

${ }^{26}$ Il ne parvint pas en revanche à faire modifier la politique du parti à l'égard de l'OTAN.

${ }^{27}$ Le SNP avait du reste fait campagne pour le Non lors du référendum de 1975 sur l'adhésion du Royaume-Uni à la CEE.

${ }^{28}$ L'indépendance en Europe allait à partir du congrès de 1988 devenir la politique phare du SNP. 
outre, opposé à toute forme de dévolution. Cet état de fait, baptisé 'Doomsday Scenario' dans la presse écossaise, mettait en évidence le déficit démocratique dont souffrait l'Écosse en raison du mode de scrutin en vigueur lors des élections au Parlement britannique.

Les résultats des élections de 1987 furent mitigés pour le SNP. Globalement le parti avait légèrement progressé en termes de pourcentage de voix et de sièges par rapport à $1983^{29}$. Les nationalistes parvinrent à reconquérir trois circonscriptions, mais ils perdirent l'élection dans les deux circonscriptions qu'ils avaient réussi à conserver en 1979. Cependant, l'impopularité des politiques gouvernementales mises en œuvre en Écosse par le gouvernement conservateur, d'une part, et l'impuissance des élus travaillistes en Écosse, pourtant très largement majoritaires, d'autre part, apportèrent de l'eau au moulin des nationalistes.

Dans ce contexte politique de déficit démocratique, le nouvel impôt local institué par le Local Government Act 1988, et baptisé poll tax par ses détracteurs, fut incontestablement l'élément qui permit au SNP de s'affirmer comme le défenseur des intérêts de l'Écosse. Le Parti travailliste, en effet, quoique hostile au nouvel impôt, était divisé sur l'attitude à adopter pour essayer d'empêcher sa mise en place. En tant que parti alternant au pouvoir à Londres avec le Parti conservateur, et donc appelé à l'avenir à gouverner, le Parti travailliste ne souhaitait pas initier une campagne massive de désobéissance civile en appelant les Écossais à refuser de s'acquitter de la poll tax, même si une telle prise de position était souhaitée par certains militants travaillistes regroupés depuis le congrès écossais du parti de mars 1988 au sein d'un groupe néo-nationaliste baptisé Scottish Labour Action. Le SNP, quant à lui, avait lancé une campagne de non-paiement pour contester la légitimité du nouvel impôt local. Or cette campagne contribua très largement à la victoire du SNP lors de l'élection partielle de Glasgow Govan, fief travailliste, le 10 novembre 1988, la première victoire du parti dans une élection partielle depuis 1973. Le candidat nationaliste, Jim Sillars, qui avait centré sa campagne sur l'opposition ferme au paiement de la poll tax et sur l'impuissance des députés travaillistes à Westminster rebaptisés par le SNP les 'feeble fifty' ${ }^{30}$, recueillit $50 \%$ des suffrages.

Malgré la victoire de Glasgow Govan, et la montée du parti dans les sondages au cours des mois qui suivirent, jusqu'à recueillir $32 \%$ des intentions de vote au mois de janvier 1989, la question de la position du SNP face à la dévolution allait une fois encore diviser le parti et nuire à sa crédibilité politique. Le parti avait en effet annoncé, lors de la publication du rapport de la CSA intitulé A Claim of Right for Scotland, au mois de juillet 1988, dans lequel l'association préconisait la mise en place d'une Convention constitutionnelle écossaise chargée de rédiger un projet d'assemblée, qu'il participerait à cette Convention. Toutefois, forts de leur succès dans l'élection partielle de Glasgow Govan, et de leur progression dans les sondages, les nationalistes entendaient bien imposer des conditions à leur participation, notamment sur le nombre de sièges attribués à chaque parti. Par ailleurs, ils voulaient être certains que la Convention n'allait pas se limiter à

${ }^{29}$ Le SNP recueillit $14 \%$, soit un peu plus de deux points de plus, et trois candidats élus, soit un de plus, qu'en 1983.

${ }^{30}$ Les travaillistes détenaient en effet 50 des 72 sièges que comptait l'Écosse aux Communes. 
cautionner un projet de dévolution similaire à celui qui avait été proposé par le Parti travailliste à la fin des années 1970. Enfin, ils exigeaient la tenue d'un référendum dans lequel le peuple écossais aurait à se prononcer sur trois options constitutionnelles possibles, à savoir, le statu quo, la dévolution et l'indépendance au sein de la CEE.

Finalement, après avoir été partie prenante dans les réunions préparatoires à la mise en place de la Convention, notamment celle du 27 janvier 1989, les instances dirigeantes du SNP décidèrent le 4 mars 1989 que le parti ne participerait pas à la Convention. Les conséquences de cette décision promettaient cependant d'être lourdes pour les nationalistes, qui apparaissaient clairement désormais comme les seuls responsables de l'échec d'une coopération qui aurait pu marquer la naissance d'un véritable front anti-gouvernemental en Écosse. Le nouveau leader du SNP, Alex Salmond, élu à la tête du parti au congrès de septembre 1990, allait donc avoir fort à faire pour effacer l'image d'intransigeance associée à son parti. Toutefois, malgré des débuts difficiles à la tête du SNP où son autorité et l'intégrité du parti furent à plusieurs reprises mises en question, les efforts déployés par le numéro un des nationalistes lui permirent finalement d'engager son parti sur la voie de la modération et d'obtenir la consécration en tant qu'architecte de la rénovation du parti lors du congrès de Perth de 1995.

\section{Le triomphe du pragmatisme et de la modération}

Malgré la popularité de l'indépendance en Europe et la victoire de Glasgow Govan, lorsque Alex Salmond devint le numéro un du SNP, le parti accusait déjà une baisse sensible dans les sondages: après un bon résultat aux élections européennes du mois de juin 1989, au cours desquelles le SNP avait obtenu 25,6 \% des suffrages, $22 \%$ seulement des Écossais lui accordaient leur soutien au mois de septembre 1990. Un an plus tard, le SNP ne recueillait plus que $19 \%$ des intentions de vote des Écossais. L'année 1991 ne fut effectivement pas une bonne année pour le SNP : non seulement il n'allait plus bénéficier de l'impopularité de Margaret Thatcher, qui avait démissionné de ses fonctions de Premier ministre au mois de novembre de l'année précédente, mais à compter du mois d'avril 1991, il perdait également l'un de ses principaux thèmes de campagne contre le gouvernement, à savoir le retrait de la poll tax, puisque John Major avait annoncé la mise en place d'un nouveau système d'impôts locaux à partir d'avril 1993. Par ailleurs, la victoire du candidat libéral-démocrate lors de l'élection partielle de Kincardine and Deeside, le 7 novembre 1991, suite au décès du député conservateur Alick Buchanan-Smith, hissait les libéraux-démocrates au rang de deuxième parti en Écosse en termes de députés élus.

Finalement le refus du SNP de participer à la Convention constitutionnelle était d'autant plus préjudiciable aux nationalistes qu'il y avait de nombreux points communs entre les propositions du SNP et celles de la Convention en matière de réforme du statut constitutionnel de l'Écosse ${ }^{31}$. Alex Salmond était considéré comme

${ }^{31}$ La Convention dévoila publiquement son premier projet d'assemblée intitulé Towards Scotland's Parliament le 30 novembre 1990. Cinq ans plus tard, jour pour jour, un second 
l'architecte de la stratégie gradualiste de l'aile gauche du parti. Même s'il restait fidèle à la position adoptée par le parti face à la Convention constitutionnelle, il souhaitait rapprocher son parti des autres partis d'opposition en Écosse une fois les élections législatives passées. La victoire du Parti conservateur lors des élections législatives du 9 avril 1992 fut pour le moins déconcertante pour les Écossais qui, se basant sur les prévisions des instituts de sondage qui donnaient le Parti travailliste vainqueur, n'avaient nullement envisagé la répétition du fameux 'Doomsday scenario'. Les résultats du scrutin furent particulièrement décevants pour le SNP, qui malgré une progression de 7,5 points par rapport aux élections de 1987, ne parvint pas à remporter un siège de plus ${ }^{32}$. La quatrième victoire consécutive des conservateurs suscita une vague de protestation en Écosse qui donna lieu à la mise en place de plusieurs groupes de pression qui revendiquaient le droit de l'Écosse à l'auto-détermination et réclamaient à ce titre un référendum sur l'avenir constitutionnel de l'Écosse.

Le mouvement de protestation atteignit son apogée avec la signature par les trois partis de l'opposition parlementaire écossaise lors du grand rassemblement du 12 décembre 1992 à Édimbourg de la «déclaration pour la démocratie » (Declaration for Democracy) demandant le rétablissement du Parlement écossais, au moment même où se tenait justement dans la capitale écossaise le sommet européen des 11 et 12 décembre 1992. La décision du SNP de s'unir cette fois, contrairement à ce qui s'était produit en 1989 lors de l'annonce de son retrait de la Convention, aux deux autres partis pour faire front contre le gouvernement conservateur de John Major était le signe indéniable d'une avancée de la part des nationalistes sur la voie de la modération. Certes, les fondamentalistes étaient toujours influents, en particulier au sein du NEC : c'était en effet sous leur pression que le parti avait adopté le slogan 'Free by '93' pour la campagne législative de 1992, mais les résultats des élections semblaient avoir donné raison aux gradualistes qui souhaitaient voir le parti revenir à plus de réalisme dans ses prétentions.

La coopération entre les trois partis d'opposition en Écosse fut de courte durée cependant. Alors que l'occasion de la tenue du sommet européen d'Édimbourg avait permis de réunir l'opposition écossaise, ce fut justement à propos de la question de la place de l'Écosse dans l'Europe que le fragile consensus entre travaillistes et nationalistes fut détruit. En effet, au printemps 2003, dans le cadre des débats sur le projet de loi gouvernemental visant à la ratification du traité de Maastricht, les nationalistes votèrent avec le Parti conservateur contre un amendement proposé par le Parti travailliste concernant le futur Comité des régions. Or il fut découvert par la suite que les conservateurs et les nationalistes avaient agi de concert, ayant passé un «accord» sur la représentation de l'Écosse au sein du Comité des régions ${ }^{33}$. Alex

projet était publié, sous le titre Scotland's Parliament. Scotland's Right, dont le projet travailliste de 1997 était très largement inspiré.

${ }^{32}$ Avec $21,5 \%$ des suffrages, le SNP n'eut que trois candidats élus.

${ }^{33}$ Margaret Ewing, chef de file du groupe parlementaire des nationalistes écossais, avait en effet écrit une lettre au Secrétaire d'État pour l'Écosse, Ian Lang, dans laquelle elle demandait au gouvernement d'octroyer à l'Écosse entre six et huit sièges sur le total des 24 alloués au Royaume-Uni au sein du Comité des régions, en échange de quoi les députés nationalistes lui apporteraient leur soutien sur la ratification du traité de Maastricht. En l'occurrence six sièges 
Salmond qui, depuis son élection à la tête du SNP, n'avait cessé d'attaquer le Parti conservateur au pouvoir, qu'il accusait d'être indifférent sinon hostile à l'Écosse, dut alors faire face à de sévères critiques de la part des membres du Comité exécutif national comme des militants, et sa position de leader en fut un temps affaiblie.

Il était pourtant essentiel, à un an des élections européennes et des élections régionales de 1994, de couper court aux dissensions et de mobiliser à nouveau toutes les forces du parti afin de conquérir de nouveaux sièges dans les deux niveaux de gouvernance. L'attaque du numéro un des nationalistes contre l'introduction par le gouvernement de la TVA sur les combustibles domestiques répondait en grande partie au besoin urgent de réparer l'erreur de l'accord passé avec le gouvernement sur le Comité des régions. Mais cette mesure gouvernementale, qui pénalisait de fait les habitants des régions les plus froides, dont l'Écosse, fournissait également au parti un thème de campagne à même de faire l'unanimité au sein du SNP, puisqu'il s'agissait de défendre les intérêts de l'Écosse contre la politique gouvernementale.

Par ailleurs, il était fondamental pour Alex Salmond de repositionner le SNP clairement au sein de l'opposition écossaise. Aussi le numéro un du SNP choisit-il de s'adresser directement aux Écossais, afin de s'expliquer sur les erreurs passées de son parti et également de leur présenter son projet politique. Les six discours qu'il prononça au cours de l'été $1993^{34}$ dans six localités différentes développaient des thèmes chers au parti, ayant trait notamment à la protection des plus vulnérables ${ }^{35}$. En outre, le leader nationaliste accusait les conservateurs de privilégier l'Angleterre au détriment de l'Écosse, à des fins purement politiques, et reprochait aux travaillistes leur incapacité à protéger les intérêts de l'Écosse. Il préconisait donc sans équivoque l'indépendance de l'Écosse au sein de la Communauté Européenne, qui seule pouvait permettre d'appliquer aux problèmes spécifiques de l'Écosse des solutions élaborées en Écosse par les Écossais. Enfin, et surtout, Alex Salmond tirait les leçons des élections législatives de 1992 et reconnaissait que son parti avait fait preuve d'un excès de confiance, notamment en adoptant le slogan Free by '93, et qu'il n'avait pas réussi à convaincre les Écossais de la valeur de son projet d'indépendance en Europe. Il était nécessaire notamment de mettre un terme à l'attitude négative, répandue dans la population et dans les rangs des nationalistes, qui consistait à rendre les Anglais responsables de tous les maux dont souffrait l’Écosse.

La mise au point effectuée par Alex Salmond durant l'été 1993 ne fut pas sans déclencher des réactions hostiles à son égard de la part de certains responsables, et aussi de certains militants, qui lui reprochaient notamment de ne pas consulter le parti avant d'agir. Mais finalement, en l'espace de deux ans Alex Salmond parvint à donner au SNP l'image d'un parti qui, sans renoncer à son ambition de mettre en

étaient promis à l'Écosse, dont quatre répartis sur la base des partis représentés en Écosse, ce qui garantissait un siège au SNP.

${ }^{34}$ Discours prononcés entre le 2 juillet et le 17 septembre 1993 et publiés par le service des publications du SNP dans un recueil intitulé Horizons without Bars.

${ }_{35}$ Parmi ces thèmes figuraient donc l'opposition à l'introduction de la TVA sur les combustibles domestiques, mais aussi la lutte contre l'extrême pauvreté et le droit de chacun à un logement correct. 
œuvre son projet politique dans une Écosse accédant au statut d'État indépendant, faisait toutefois preuve de réalisme quant aux conditions nécessaires et aux moyens à sa disposition pour atteindre son objectif. Outre les bons résultats obtenus aux élections régionales du 4 mai 1994, puis aux élections européennes du 13 juin suivan $^{36}$, le numéro un du SNP pouvait également se satisfaire de la bonne tenue de son parti dans les sondages, puisque, depuis le mois d'octobre 1992, le SNP arrivait constamment en deuxième position, devant le Parti conservateur et les libérauxdémocrates ${ }^{37}$.

L'unité du parti sur la stratégie à adopter pour atteindre l'objectif politique d'indépendance était cependant toujours loin d'être acquise. L'aile fondamentaliste était finalement satisfaite de ce que la découverte de l'accord passé par le groupe parlementaire avec le gouvernement conservateur avait mis fin à l'amorce de coopération entre les travaillistes et les nationalistes. Mais dans la perspective des élections législatives suivantes, prévues au plus tard pour le printemps 1997, et d'une victoire des travaillistes, se posait à nouveau la question du choix de la meilleure stratégie à adopter pour le SNP face au projet de parlement écossais inscrit au programme politique du Parti travailliste. Aussi les propos tenus par Alex Salmond au cours d'un entretien accordé au journal du dimanche Scotland on Sunday, publiés le 28 janvier 1995, étaient-ils de nature à provoquer la colère de l'aile fondamentaliste du parti. Il déclarait en effet en substance qu'en cas de victoire travailliste, il lui paraissait souhaitable d'envisager de façon positive la mise en place d'un parlement écossais, comme un tremplin vers l'indépendance. Finalement, alors qu'il n'était pas prévu de débattre de la position du parti face à la dévolution, cette question domina le congrès annuel de 1995 à Perth, et malgré la tentative de la part de l'aile fondamentaliste de faire adopter son point de vue comme politique officielle du parti, le congrès consacra le triomphe de la modération et du pragmatisme incarnés par le leader.

À moins de deux ans de nouvelles élections législatives, la maturité acquise par le SNP depuis celles de 1992 ne faisait aucun doute : tout d'abord le temps des slogans jusqu'au-boutistes semblait bel et bien révolu. Par ailleurs, conscients de l'importance de préparer le passage de l'Écosse au statut d'État indépendant, les nationalistes mirent en place au mois de janvier 1995 un groupe de travail baptisé 'Highway to Independence', chargé d'étudier les implications pratiques de la dissolution de l'Union et les modalités à mettre en œuvre pour l'accession à l'indépendance.

\footnotetext{
${ }^{36}$ Lors des élections régionales, le SNP, avec 73 élus sur un total de 534 pour toute l'Écosse, se plaçait en deuxième position devant les libéraux-démocrates (64 élus) et les conservateurs (31 élus). Par ailleurs, le SNP avait recueilli 32,6\% des voix aux élections européennes du 13 juin (7 points de plus qu'en 1989), et surtout le parti comptait désormais deux élus sur les huit dont disposait l'Écosse au Parlement de Strasbourg.

${ }^{37}$ Toutefois, l'écart qui séparait le SNP du Parti travailliste rendait plus qu'improbable la perspective de voir le SNP remporter plus de la moitié des sièges aux élections législatives suivantes.
} 


\section{Conclusion}

Les élections législatives du $1^{\text {er }}$ mai 1997 resteront longtemps associées en Écosse à deux faits sans précédents dans l'histoire de l'Union : d'une part, la perte pour le Parti conservateur de tous ses sièges écossais, et d'autre part, le déclenchement du processus de réforme de la constitution. Les résultats obtenus par le Parti nationaliste étaient une fois encore mitigés : le parti n'avait pas globalement progressé en termes de pourcentage de voix recueillies par rapport aux élections législatives de 1992; en revanche grâce à une campagne ciblée pour les circonscriptions dans lesquelles le parti était à même de remporter l'élection, il doublait le nombre de ses députés à Westminster (6 en tout). Ses trois candidats sortant étaient réélus, mais le parti n'était pas parvenu à battre le Parti travailliste dans ses fiefs de la Central Belt ${ }^{38}$.

L'attitude des nationalistes au lendemain des élections laissait présager une coopération possible dans la campagne du référendum, même si la position officielle du parti ne pouvait logiquement être arrêtée avant la publication par le gouvernement de son livre blanc sur le futur Parlement écossais ${ }^{39}$. De fait après réunion du comité exécutif national du parti, le 27 juillet, le SNP annonça qu'il se joignait aux travaillistes et aux libéraux-démocrates pour faire campagne pour un double Oui dans le référendum du 11 septembre 1997. Cette campagne restera exemplaire dans l'histoire du mouvement pour le Home Rule en Écosse, de par la décision des trois partis favorables à une réforme du statut de l'Écosse au sein du Royaume-Uni d'unir leurs forces pour défendre la mise en place d'un parlement écossais, et ce malgré leurs divergences de vue sur la finalité du projet de dévolution proposé par le gouvernement travailliste. Une fois la mise en place d'un parlement écossais acquise, les trois partis allaient cependant redevenir des adversaires pour les premières élections générales écossaises; l'heure était donc de nouveau à l'affrontement entre les deux principaux rivaux dans la course au pouvoir à Édimbourg, à savoir le Parti travailliste et le SNP. À l'issue des élections du 6 mai 1999, le Parti travailliste conclut un accord avec les libéraux-démocrates pour former le nouvel Exécutif écossais, tandis que le SNP, consacré comme deuxième force politique en Écosse en termes de suffrages et aussi, grâce au scrutin proportionnel, en termes de sièges, devenait l'opposition officielle au Parlement écossais.

\footnotetext{
${ }^{38}$ Le SNP arrivait toutefois en deuxième position dans 43 circonscriptions sur 72.

${ }^{39}$ Le Livre blanc intitulé Scotland's Parliament fut publié le 16 juillet 1997.
} 